\title{
Kai Sammet
}

\section{Wozu man die Schizophrenie gebrauchen kann: Semantik, Pragmatik, Offentlichkeit(en) zwischen ca. 1910 und 1930}

\author{
Schizophrenie „mit Abwandlungen“
}

Der Heidelberger Psychiater Karl Wilmanns bemerkte 1922, die Geschichte der Dementia praecox sei die Geschichte der Psychiatrie der letzten dreißig Jahre. Darf man das wörtlich nehmen? Sicher war diese Aussage auch Rhetorik: Wilmanns referierte auf der Jahrestagung des „Vereins für Psychiatrie“ über die Entwicklung der Dementia praecox-Lehre und rechtfertigte mit seiner Formulierung, dass er deren Geschichte nur in "Umrissen zeichnen“ könne. ${ }^{1}$ Dennoch: Gesagt ist gesagt. Die Dementia praecox wäre demnach also nicht nur das pochende Herz der Disziplin, sondern diese selbst. Wilmanns wollte die Bedeutung der Schizophrenie betonen; seine Formulierung hat semantischen Mehrwert. Gesagt ist gesagt, und: gesagt ist getan. Wilmanns tat etwas mit dieser Aussage, er gebrauchte sie für einen Zweck, und er gebrauchte das Konzept der Schizophrenie für einen Zweck. Und darauf kommt es an: Er deutete nicht, was Schizophrenie ist, sondern er verwendete dieses Konzept umgekehrt zur Deutung dessen, was Psychiatrie ist. Mir geht es im Folgenden um Gebrauch und Pragmatik der semantischen Konstruktionen der Schizophrenie als Deutungsfolien für anderes. Im bunten Strauß antirealistischer Epistemologien ist der Konstruktivismus nur die derzeit duftendste Blüte. Er untersucht die „Gemachtheit“ von Konzepten. ${ }^{2}$ Bildlich gesprochen: Konstruktivisten interessiert, wie ein Hammer hergestellt wird. Was aber, wenn ein Konzept „verfertigt“ ist? Wird es petrifiziert, stets gleich verwendet, an andere Orte, in andere Kontexte transferiert - wie ein Tisch, der stets derselbe bleibt, unabhängig davon in welcher Wohnung er steht? Wörter seien „Verkehrsgut“ im Denkverkehr, so der polnische Wissenschaftshistoriker Ludwik Fleck. Ein Denkkollektiv (also: eine Öffentlichkeit) entstehe, wenn zwei Menschen Gedanken austauschten. Mitteilung von Wissen sei nicht „Translokation eines star-

\footnotetext{
${ }^{1}$ Karl Wilmanns: Die Schizophrenie. In: Zeitschrift für die gesamte Neurologie und Psychiatrie 78 (1922), S. 325-372, hier: S. 325.

${ }^{2}$ Vgl. zur Schizophrenie Brigitta Bernet: Schizophrenie. Entstehung und Entwicklung eines psychiatrischen Krankheitsbilds um 1900. Zürich 2013.
} 
ren Körpers“: Gedanken wandern vom einen zum andern, „jedesmal etwas umgeformt“, denn jeder knüpfe „andere Assoziationen an sie an“, der Empfänger verstehe den Gedanken nie genau so „wie ihn der Sender verstanden haben wollte“. 3 Logisch gesprochen handelt es sich also um Begriffsextension und -intension. Die Extension des Begriffs meint den Umfang des Begriffs: Welche Gegenstände in der Welt fallen unter ihn - nun eben alle Schizophrenen. Intension meint die Prädikate oder Attribute des Begriffs: $\mathrm{X}$ ist schizophren, wenn die Symptome $S_{1} \ldots S_{n}$ vorliegen. Semiotisch gesprochen kann man Zeichenprozesse als dauerndes Modeln im relationalen Geviert von Zeichen, Designat, Interpretant und Interpret auffassen. ${ }^{4}$ Eine Spur im Sand (Zeichen) kann auf eine Schlange hinweisen (Designat). Ein Interpret (Schlangenphobiker) bildet den Interpretanten „Gefahr, Flucht“; ein anderer Interpret (Schlangenforscher) bildet dagegen den Interpretanten „Neugier, Stehenbleiben“. Überlegungen, die Pragmatik und Gebrauch von Konzepten akzentuieren, legen eine instrumentalistische Epistemologie nahe: ${ }^{5}$ Begriffe, Theorien sind Werkzeuge. Sie sind weder (Realismus) Abbilder der Wirklichkeit, noch zielt der Instrumentalismus auf „Konstruiertheit“. Werkzeuge sind multipel verwendbar: Mit einem Hammer kann ein Handwerker Nägel in die Wand schlagen; Serienmörder benutzen ihn anders (und für anderes). Transferiert der Verbrecher ihn aus seinem ursprünglichen Verwendungsgebiet in ein anderes? Man kann Hans Prinzhorns „Bildnerei der Geisteskranken“ als „Beispiel für Transferprozesse zwischen wissenschaftlichem und außerwissenschaftlichem Wissen“, zwischen psychiatrischer „Fachdiskussion“ und „im nicht wissenschaftlichen Kontext" verbreiteter Sicht auf die Schizophrenie ansehen. ${ }^{6}$ Das ist das Tisch-Modell. Doch die Bedeutungen eines komplexen Zeichens wie Schizophrenie oszillieren immer. Spracherwerb (und die Konstruktion eines Begriffs) erfolgen in der Praxis, in spezifischen Kontexten (zum Beispiel in verschiedenen Kliniken); daher lösen Worte bei jedem unterschiedliche Assoziationen aus, das heißt Intensionen sind variabel. ${ }^{7}$ An der Heidelberger Klinik war, folgt man den Ausführungen Karl Jaspers, der dort 1908 bis 1915 tätig war, Kraepelins Psychiatrie „mit Abwandlungen“ verbindlich. ${ }^{8}$ Diagnosen, abgelesen an Kranken, zur Krankheitseinheit reifiziert, sind als semantische Konzepte nicht petrifiziert. Gab es je ein Krankheitsbild Schizophrenie? Bedeutungen wuchern, da sie gebraucht werden: Pragmatik sticht Semantik. Es gibt nicht die Schizophrenie, nur oszillierende Pluralitäten.

${ }^{3}$ Ludwik Fleck: Entstehung und Entwicklung einer wissenschaftlichen Tatsache. Einführung in die Lehre vom Denkstil und Denkkollektiv. Frankfurt a. M. 1980, S. 145, S. 58.

${ }^{4}$ Charles W. Morris: Grundlagen der Zeichentheorie. Frankfurt a. M. 1988.

${ }^{5}$ Reinhard Heede: Instrumentalismus. In: Joachim Ritter/Karlfried Gründer (Hg.): Historisches Wörterbuch der Philosophie, Bd. 4. Basel/Stuttgart 1976, Sp. 424-428.

${ }^{6}$ Doris Kaufmann: Kunst, Psychiatrie und ,schizophrenes Weltgefühl' in der Weimarer Republik. In: Matthias Bormuth/Klaus Podoll/Carsten Spitzer (Hg.): Kunst und Krankheit. Studien zur Pathographie. Göttingen 2007, S. 57-72, hier: S. 58.

7 Angela D. Friederici: Denn das Wort ist im großen Netz verborgen. In: FAZ, 19. 5. 2014, N 2.

${ }^{8}$ Karl Jaspers: Philosophische Autobiographie. München 1977, S. 20. 
Was hat das mit Öffentlichkeit(en) zu tun? Ich skizziere drei Beispiele, in denen Schizophrenie-Semantiken zur Deutung von Wahn und Werk Friedrich Hölderlins (1770-1843) benutzt wurden. Hölderlin erkrankte nach 1800 an einer Störung, die 1806 zur Aufnahme ins Tübinger Klinikum führte. Von Mai 1807 bis zu seinem Tod lebte er als Pflegling bei einer Handwerkerfamilie. ${ }^{9}$ Es geht im Folgenden nicht um Werk oder Krankengeschichte, sondern darum, was Autoren damit anstellten: 1909 schrieb Wilhelm Lange-Eichbaum eine Hölderlin-Pathografie, 1922 beschäftigte sich Karl Jaspers mit ihm, 1929 Alexander Mette. Zunächst zeige ich auf, wie jeder der Autoren die Schizophrenie(n) als Stoff für das Weben anderer Semantiken benutzte. Dann skizziere ich einige Konzepte von Öffentlichkeit. Es folgen verschiedene Kritikpunkte am traditionellen Öffentlichkeitsbegriff. Beides beziehe ich zurück auf meine Beispiele: Könnte die Betrachtung von Inhalten, dem Gebrauch von Schizophrenie-Semantiken, Ergänzungen zum Begriff der Öffentlichkeit(en) liefern?

\section{Wilhelm Lange: Psychiatrie sticht Philologie}

Wilhelm Lange studierte unter anderem in München Medizin; er promovierte bei Emil Kraepelin. Als Assistent Robert Gaupps in Tübingen schrieb er eine Hölderlin-Pathografie, die er dem Germanisten Franz Zinkernagel, Herausgeber der Werke Hölderlins, widmete. ${ }^{10}$

Wie las er Hölderlin? Die „Wissenschaft der Pathographie“ war eine Art Forensik: Sachverständige (das konnten nur Psychiater sein) erstellten für Literaturhistoriker Gutachten, damit diese beurteilen konnten, was an einem Werk abnorm war. Derjenige sei geistig normal, dessen Seele inneren Gesetzen der Gattung gehorche. Und das meinte, dass diese Seele ein geistiges Abbild der Außenwelt in sich bilden konnte. Psychopathen hätten ein „Zerrbild der Welt“, seien aber nicht geisteskrank (in diesem Fall käme Intelligenzminderung hinzu). Psychopathie ähnelte Nervosität, sei aber angeboren. Früh würden Lebensreize falsch verarbeitet. Ein Psychopath denke, fühle, wolle auf eine Art, die nicht zum angestrebten Zweck passe. Seine Widerstandsfähigkeit sei gering, ihm fehle die Harmonie der Person, er sei labil, widersprüchlich, unberechenbar, suggestibel (männliche Psychopathen seien unangemessen feminin, Suggestibilität passe nicht zum Mann), (zu) phantasievoll, sein Ich-Komplex sei hypertroph, Selbstzucht fehle, nur günstige Bedingungen (wie richtige Erziehung, Lenkung durch andere Personen, passende Berufswahl) verhinderten seinen Absturz.

\footnotetext{
9 Dieter Burdorf: Friedrich Hölderlin. München 2011, S. 14-29.

${ }^{10} \mathrm{Zu}$ Lange-Eichbaum und Zinkernagel: Klaus Schonauer: Germanistisch-psychiatrische Deutungsrivalität um Hölderlin in erster Instanz: Wilhelm Lange und Norbert von Hellingrath. In: Uwe Gonther/Jann E. Schlimme (Hg.): Hölderlin und die Psychiatrie. Bonn 2010, S. 140-176 (dort weitere Literatur). Zum Folgenden: Wilhelm Lange: Hölderlin. Eine Pathographie. Stuttgart 1909.
} 
Bei der Beurteilung spielte der Gesichtsausdruck eine große Rolle. Alle Porträts Hölderlins zeigten einen schwärmerischen Theologenjüngling: weltfremd und voll Seelenadel, aber ohne robuste Männlichkeit. Er habe Hang zum Abstrakten, aber kein Talent dafür gehabt, seine Begriffe seien diffus, er neige zur Weltflucht, habe schöne Verse machen wollen. Die Basis der Psychopathie liege in seinen Gefühlen, er habe Affektauslenkungen gehabt, rauschhafte Gefühle, ihm fehlte Realitätssinn. Gehörte all das nicht zur Romantik? Romantiker liebten Halbdunkel und Katholizismus. Psychopathie und Romantik seien eins. Romantische Literaturbewegungen seien von Psychopathen begründet, denen psychisch-epidemisch viele Psychopathen folgten, durch Suggestion würden gar Normale mitgerissen. Auf psychopathischer Basis habe Hölderlin Depressionen entwickelt, Ausdruck einer Verflechtung von Umwelt und Anlage. Hölderlin sei nicht nur Opfer der Umstände, vieles sei Anlage gewesen. Manches entspreche auch einem typischen Künstlerschicksal, nicht seiner Psychopathie.

Die sichtbaren Symptome der Geisteskrankheit vervollständigten das lückenhafte Bild. Hölderlin, so Lange, litte an einer Katatonie, die kraepelianisch beschrieben wurde: Sie wurde von ihm verstanden als eine Unterform der Dementia praecox, gekennzeichnet durch Beginn im frühen Lebensalter und Ausgang in Blödsinn. Die Wissenschaft habe durch Beobachtung von Entstehung, Verlauf und Ausgang eine natürliche Krankheitseinheit entdeckt, einen Abbauprozess, in $70 \%$ der Fälle ließe sich eine Hirnveränderung feststellen. Kranke halluzinierten, ihre Aufmerksamkeit sei gestört, sie seien leicht ablenkbar oder blieben an einer Vorstellung haften (Sperrung). Normales, von Leit- und Zielvorstellungen beherrschtes Denken ginge verloren, es käme zu Zerfahrenheit, Manieriertheit, die Steuerung fehlte. Darunter litten besonders differenziertere ethische und ästhetische Regungen: Lange hatte noch nie künstlerisch Relevantes von Schizophrenen gesehen. Willenssperrung und Negativismus waren Ausdruck katatoner Bewegungsstörungen, immer mangelte es an Schönheit und Anmut. In Gedichten fand man völlig leere Banalitäten. Am Ende blieb gewissermaßen ein ausgebrannter Krater zurück.

Bei Hölderlin fehlten (fremd-)anamnestische Daten. Doch seine Kunst sei, so Lange, ein präziser Seismograph seiner Leistungen. Demnach habe er 1802 einen ersten Schub erlitten: Transparenz und Struktur des Denkens seien geschwunden, ebenso das Stilgefühl sowie das Gefühl für den Unterschied zwischen Dichtungsund Alltagssprache. Lange entdeckte seltsame Wortkomposita. In „Der Gang aufs Land. An Landauer“ fielen substantivierte, abstrakte Adjektive auf. ${ }^{11}$ Hatte Hölderlin Halluzinationen gehabt? Stellen in „Patmos“ sprächen dafür („Im Zorne sichtbar sah ich einmal/ Des Himmels Herrn“). ${ }^{12}$ Die freien Rhythmen einiger später Gedichte erschienen nicht als Fortschritt, sondern wurden als Ausdruck

11 Vgl. z. B. Friedrich Hölderlin: Der Gang aufs Land. An Landauer, Z. 1: „Komm! ins Offene, Freund! zwar glänzt ein Weniges heute/Nur herunter“. Abgedruckt in: Friedrich Hölderlin: Sämtliche Gedichte. Text und Kommentar. Hg. von Jürgen Schmidt. Frankfurt a. M. 2005, S. 276.

12 Hölderlin: Gedichte (wie Anm. 11), S. 355, Z. $171 \mathrm{f}$. 
sinkender Formbeherrschung bewertet. In „Andenken“ fand Lange Geschraubtes („noch denket das mir wohl“ für: „ich kann mich erinnern“). Das Weglassen des Artikels in „Patmos“ war seiner Meinung nach manieriert, das Gedicht insgesamt wirr. Man erahne manchmal Sinn, jeder würde aber „sagen: das verstehe ich nicht.“ Hölderlins Intelligenz - davon war Lange überzeugt - sank, auch wenn er nicht völlig „verblödete“. Während seines Klinikaufenthalts erhielt er Mittel gegen die „Torpidität“ des Nervensystems - das durfte man als katatonischen Stupor übersetzen -; durchgehend also deutete Lange Hölderlins Sprache und Verhalten retrospektiv mithilfe der psychiatrischen Diagnostik um 1900.

Die Lange zugänglichen Urteile über Hölderlins Wahn waren ernüchternd: Drei Ärzte hatten Hölderlin „begutachtet“, nur einer von ihnen war kein Psychiater. Genau dieser stellte bei dem Dichter auch keine Psychose fest. Lange ließ jedoch nur die psychiatrischen Gutachten gelten. Für ihn war klar: Hölderlin war ein Psychopath. Als solcher hatte er sein Werk geschaffen; er wurde später geisteskrank, aber mit seiner dichterischen Leistung hatte die Katatonie nichts zu tun.

Es ist leicht, Argumente gegen Lange vorzubringen. Er machte Fehler. ${ }^{13} \mathrm{Er}$ spielte die Romantik gegen die Klassik aus. ${ }^{14}$ Er wollte ,gemeinverständlich ‘ sein und diese Popularisierung erforderte eine Reduktion von Komplexität. Diese Aufgabe löste Lange durch vereinfachende Vereindeutigung: ${ }^{15}$ Er leimte Hölderlins Person aus Stereotypen zusammen; das Ergebnis war ein Hybrid aus romantischem Jüngling, Décadent und Massenpsychologiediskurs.

\section{Alexander Mette: Sturm und expressionistische Wortkunst}

Mette ging es um die Sprache Schizophrener, die er als eindrucksvolles Symptom betrachtete. Sprache - davon war er überzeugt - ließ in die Psyche des Sprechers blicken. ${ }^{16}$ Poetik untersuchte „Formeigentümlichkeiten“ der Sprache, fragte nach psychischen Bedingungen dichterischen Schaffens; somit könne man auch pathologische Sprache mittels Poetik untersuchen. Gab es gemeinsame Grundlagen für Dichtung und Psychose? Fand in der Krankheit eine psychische Umwandlung statt, deren Ergebnis den seelischen Grundlagen kreativer Prozesse ähnelte?

13 Als Beispiel hierfür: „noch denket mir das wohl“ ist nicht verschroben, also Krankheitssymptom, sondern ein dialektaler schwäbischer Ausdruck für ,ich erinnere mich daran“; Hölderlin: Gedichte (wie Anm. 11), S. 1021.

${ }^{14}$ Die Beziehungen zwischen Klassik und Romantik sind komplex; Lange nimmt erstens Goethes Aussage, das Klassische sei das Gesunde, das Romantische krank, zur Vorlage; vgl. Stefan Greif: Arbeitsbuch Deutsche Klassik. Paderborn 2008, S. 91. Zweitens folgt er Vorstellungen über Regelmäßigkeit, Vollkommenheit und Harmonie; vgl. dazu: Matthias Buschmeier/Kai Kauffmann: Einführung in die Literatur des Sturm und Drang und der Weimarer Klassik. Darmstadt 2010, S. 61.

${ }^{15}$ So „übersetzte“ sich Lange die „Torpidität“ des Nervensystems, wegen derer Hölderlin in Tübingen Medikamente erhielt, unreflektiert als katatonen Stupor.

${ }_{16}$ Zum Folgenden vgl. Alexander Mette: Über Beziehungen zwischen Spracheigentümlichkeiten Schizophrener und dichterischer Produktion. Dessau 1929. 
Konnten sich Schizophrenie und Kreativität gegenseitig erhellen? Das waren interessante Fragen bezüglich der Deutungsmuster psychischer Krankheit. Hier fungierte die Schizophrenie als Deutungsfolie für die Quellen des Kreativen. Walter Morgenthaler, Entdecker des schizophrenen Künstlers Adolf Wölfli, glaubte 1921, dass die dem formalen Aufbau eines Bildes inhärenten Prozesse sichtbar würden: Die Schizophrenie zerlegte Denkfunktionen und machte so deren Verbindung im Gesunden deutlich. ${ }^{17}$ Die Schizophrenie fungierte gewissermaßen als Mikroskop zur Erkennung der Makro-Phänomene in Bildern. Das ist in doppeltem Sinn ein Deutungsmuster der Schizophrenie: Als Krankheit zeigt sie die Mikromorphe des Denkens; zugleich war sie Deutungsressource in Kunsttheorie und -psychologie insgesamt. Eine weitere Funktion zeigt sich, eine Art ReboundEffekt. Erkenntnisse über Kreativität fördern das Wissen über die Schizophrenie. Mette behauptete, dass hinter der Ähnlichkeit von Krankheit und Kunst ein „verzweigtes Nebeneinander“ einer „einheitlichen Gesamterscheinung“ stecke.

In drei Schritten verband er Schizophrenie und Expressionismus. Er erörterte Schriften eines Patienten, schob Hölderlin in dessen Nähe und schließlich, conclusio, verwob er die Ergebnisse mit expressionistischer Wortkunst.

1. In den Schriften eines 25-jährigen Patienten gab es neben durch die Krankheit verursachten kognitiven Defekten auch Dichtung und Philosophie. Mette diagnostizierte eine klare Syntax, mustergültige Hypotaxe und Gliederung im Einzelsatz. Der Patient hatte ein gut entwickeltes und erhaltenes Sprachgefühl, das Wortmaterial war nicht bizarr, sondern kreativ, der Patient achtete auf Sprache, benutzte oft Abstrakta zum Ausdruck von Seelischem, umständlich-intensives Formulieren war Ausdrucksnot und Ausdrucksfähigkeit, er wollte exakt sein. So erschienen Neologismen sinnvoll, nicht krankhaft. Der Patient besaß so Mette - psychologisches Gespür. In einem lyrischen Text fand Mette die „Pathetik dichterischer Erregung“. Genau dies - Stärke emotionalen Erlebens - sahen Poetologen als Wurzel des Schöpferischen.

2. Dann lobte Mette Hölderlins Neuinterpretation durch Norbert von Hellingrath, Germanist aus dem George-Kreis und Herausgeber des Hölderlin'schen Spätwerks. Die kaiserzeitliche Germanistik hatte dieses als krank gewertet. Besonders von Hellingrath leitete einen „Paradigmenwechsel“ ein. ${ }^{18} \mathrm{Er}$ habe, so Mette, eine nachvollziehbare kontinuierliche Entwicklung Hölderlins bis 1805 bewiesen: Hölderlin gebe Gedachtes präzis wieder, benutze „kühne Komposita“. Dass von Hellingrath Krankheitsniederschläge aus kunsttheoretischer Sicht beurteilte, war für Mette Beleg dafür, dass Schizophrenie und Kreativität eng verwandt waren. Somit ließ sich Schizophrenes auch kunsttheoretisch erklären. Wie sein Patient habe auch Hölderlin oft jene Grenze berührt, an der das mit subjektivem Sinn "gefüllte Wort objektiv nicht ganz zureicht“. Mette benötigte weitere Patientenäußerungen für seine Argumentation. Hier

17 Walter Morgenthaler: Ein Geisteskranker als Künstler. Bern 1912, S. 83.

18 Vgl. dazu: Henning Bothe: ,Ein Zeichen sind wir, deutungslos ${ }^{`}$ - Die Rezeption Hölderlins von ihren Anfängen bis zu Stefan George. Stuttgart 1992. 
griff er auf die Spracherzeugnisse einer Patientin (bei der die Syntax gelitten hatte) zurück. Fragile Syntax, „Haften am Anschaulichen und komplexen, symbolischen Charakter der Begriffsbildung“ zeigten für Mette erhöhte Emotionalität; das war der Grundunterschied zwischen Dichter und Durchschnittsmensch. Bei der Kranken standen Sätze „wie reichbewegte“, aber ziellose Semantiken nebeneinander. Der Gedankenfaden riss ab. Der Subjektivismus der Patientin, ihr einseitiger Ausdrucksdrang behinderte das Verständnis. Sprache besaß - so Mette - eine Ausdrucks- und eine Mitteilungsfunktion. Bei Schizophrenie finde sich ein Plus an Ausdruck, ein Minus an Kommunikation. Schizophrene neigten zum Rhythmus, daraus resultiere ihr Hang zur Lyrik. Nun war rhythmischer Stil aber auch charakteristisch für einen bestimmten gesunden psychischen Typus, der für seine Stärke emotionalen Erlebens bekannt war. Wenn das so war, dann war, was Schizophrene produzierten, nicht pathologisch, denn das gab es ja auch bei einem gesunden Typus.

3. Mette stellte ein Gedicht August Stramms einem Gedicht eines Schizophrenen gegenüber und entdeckte Ähnlichkeiten: Das Gedicht folgte Gesetzen der Wortkunst des Sturmkreises Herwarth Waldens. Dass das kein Zufall war, bewies die Theorie expressionistischer Lyrik. Demnach drückten Begriffe das Erleben von Ungreifbarem aus. Jeder Begriff war komplex; für ihn „die Sprachtongestalt“ zu finden, war die Aufgabe des Dichters als „Wortschöpfer“. Die „Wortgestalt als Einzelwort“ konzentrierte den Begriff in ein Wort. Das führte zur Verkürzung: Man ging zurück zum Stammwort (bären für gebären); Deklinations- wie Flexionsendungen und Artikel (wie bei Hölderlin) wurden weggelassen. Aus Wortverkürzung wurde Wortveränderung: Verben wurden substantiviert, Substantive zu Verben („Kind“: „kinden“). Im Satz erfolgte Verkürzung durch Weglassen von Präposition, Kopula; transitive Verben wurden intransitiv („Die Bäume und Blumen blühen“ wurde zu „Baum blüht Blume“). ${ }^{19}$ Verknappungstechniken der Wortkunst gingen auf Kandinsky und den Futurismus zurück. Ein Wortkunstwerk war ein der Musik ähnliches „Sprachtonwerk“. Also spielten Klang und Rhythmus eine Rolle, aber auch Abstraktion als Ausdruck großer Beunruhigung. Diese Sprache sollte nicht denotativ sein, sondern "Wahrnehmung, Vorstellung und Empfindung in einen rhythmischen Fluss“ bringen und auf dahinterstehende Mächte verweisen. ${ }^{20}$ Diese Techniken tauchten als zweckvoll auch bei Schizophrenie auf; die Gleichheit innerer Bedingungen machte sie verwandt mit dem Expressionismus. Mette näherte Schizophrenie und Dichtung noch weiter an mittels der ungelenken Äußerungen eines Imbezillen, die frühen Stufen emotionalen Lebens ähnelten. Schizophrene wiederholten magisch-symbolisches Denken „Primitiver“ oder von Kindern: Festgestellt wurde erneut ein Plus an Emotion und Ausdruck, ein Mangel an Kognition und Kommunikation. Die Schizophrenie bewirke - so Mette - eine Re-

${ }_{19}$ Vgl. zum Folgenden: Thomas Anz: Literatur des Expressionismus. Stuttgart/Weimar ${ }^{2} 2010$, S. $157-160$.

${ }^{20}$ Frank Krause: Literarischer Expressionismus. Paderborn 2008, S. 158-160. 
gression. Diese sei Ausdruck des Abbaus, der Seelisches auf frühere Entwicklungsstufen bringen würde. Zugleich steigerten sich einige Leistungen, die Krankheit setze auch produktive Kraft frei.

Auch gegen Mette lassen sich Einwände finden; hier sei nur die Gesamttendenz erwähnt. Er wählte für seine Verbindung von Schizophrenie und Wortkunst Passendes aus, versteckte Widersprüche, rückte Schizophrenie, Hölderlin und Wortkunst-Konstruktivismus so lange aneinander, bis sie sich leicht ineinander spiegeln ließen. Es überrascht nicht, dass Hölderlin von Mette zum Vorläufer der Wortkunst erklärt wurde.

\section{Karl Jaspers: Philosophie - Existenz und Weltanschauung}

Jaspers verstand Schizophrenie nicht à la kraepelienne wie Lange. Seines Erachtens war der Begriff der Schizophrenie mehrdeutig. Erstens ging er davon aus, dass es sich bei der Schizophrenie um einen organischen Prozess handelte, der aber nicht Ausdruck bekannter Hirnkrankheiten war und durch den die Kranken nicht wieder ihr früheres Leistungsniveau erreichten. Zweitens verstand Jaspers unter Schizophrenie verschiedenste psychische Veränderungen. Der Prozess, der nur kausal erklärbar, und die Psychologie, die dem Verstehen zugänglich war, koinzidierten weitgehend, jedoch nicht völlig. ${ }^{21}$

Jaspers erörterte in seiner vergleichenden Pathografie verschiedene Künstler (und einen Mystiker), die ihm als Beispiele für verschiedene Spielarten der Schizophrenie erschienen. Zuerst behandelte er August Strindberg, dann Friedrich Hölderlin, Emanuel Swedenborg und schließlich Vincent van Gogh. Warum traktierte Jaspers Strindberg, obwohl er ihn nicht leiden konnte? Genau aus diesem Grund. Strindbergs Störung war zentral für dessen Existenz und Weltanschauung. Jaspers konnte zentrale Begriffe seiner Philosophie darstellen: Er empfahl als Hintergrundlektüre zu seiner Pathografie nicht nur seine „Allgemeine Psychopathologie“ (1913), sondern auch seine „Psychologie der Weltanschauungen“ (1919), ${ }^{22}$ für ihn „die früheste Schrift“ der „Existenzphilosophie“.23

Jaspers beschrieb Strindbergs „ursprüngliche Artung“24 ähnlich wie Lange Hölderlins Psychopathie, buchstabierte das aber auf existenzphilosophisch. Strindberg sei empfindlich, egozentrisch, andererseits unsicher, disharmonisch. Ihm fehle „synthetisches Vermögen“. Die Schizophrenie habe 1882 unspezifisch begonnen, 1886 sei der erste Schub aufgetreten, dann habe sie sich unter anderem mit

${ }^{21}$ Vgl. zum Folgenden: Karl Jaspers: Strindberg und Van Gogh. Versuch einer pathographischen Analyse unter vergleichender Heranziehung von Swedenborg und Hölderlin. Leipzig 1922.

22 Karl Jaspers: Allgemeine Psychopathologie. Für Studierende, Ärzte und Psychologen. Berlin 1913; ders.: Psychologie der Weltanschauungen. Berlin 1919.

${ }^{23}$ Jaspers: Autobiographie (wie Anm. 8), S. 33.

24 Jaspers: Strindberg und Van Gogh (wie Anm. 21), S. 4. 
Verfolgungsideen ausgebreitet und 1896 sei der Gipfel der Schizophrenie mit Halluzinationen erreicht worden. Es folgten paranoide Fluchten, ab 1898 sei der abgestumpfte Endzustand erkennbar gewesen.

Bei Strindberg gebe es keine sich in der gesamten Existenz entfaltende Weltanschauung, nur „formales Leben“, ohne „Idee humaner Totalität“. Was aber war bei Jaspers Existenz ? $^{25}$ Wissenschaft beziehe sich - so Jaspers - auf Gegenstände, die Philosophie nicht. Sie ziele auf Sein, das ungegenständlich ist, sie gehe methodisch anders vor als die Wissenschaft. Transzendenz und Existenz seien die eigentlichen Gegenstände der Philosophie. Sein sei Existenz, wenn es einem dadurch gegenwärtig werde, dass man es selbst sei: Man könne viel über sich wissen, dann sei man sich Gegenstand. Aber man sei für sich selbst als Ich mehr als alles gegenständlich Wissbare. Sein sei Transzendenz, wenn es gegenständlich werde als Chiffre, es bliebe jedoch nur für Existenz erfassbar. Philosophie transzendiere Gegenständlichkeit, sie transzendiere die Subjekt-Objekt-Spaltung in zwei Schritten. Im formalen Transzendieren würden wir uns vom Gegenständlichen ins Gegenstandslose aufschwingen. Dann gingen wir wieder zurück: Immanentes Transzendieren sei Rückkehr in eine veränderte Gegenständlichkeit, das bezeichnet Jaspers als Chiffren, diese seien keine sichtbar objektiven noch auch transzendente Gegenstände, sondern Verweise auf Transzendenz.

Weltanschauung sei etwas „Ganzes“, „Universales“, sie „offenbart sich in Wertungen, Lebensgestaltung“, in der „erlebten Rangordnung der Werte“, das seien „Ideen, das Letzte und das Totale des Menschen, sowohl subjektiv als Erlebnis und Kraft und Gesinnung “26 - all das fehle Strindberg. Der segele stets am Gegenwartswind, sei der geborene Literat. Bei ihm trete Formales in den Vordergrund, Zweifeln, Bekämpfen, fanatisches Behaupten, daher komme sein endloser Wechsel von Weltanschauungen. Zwar verändere die Schizophrenie seine Weltanschauung hin zum Religiösen, das habe aber nur neues Material gebracht, jedoch keine neuen Ideen. Strindbergs Antifeminismus sei keine Weltanschauung. Sein Verhältnis zu Frauen erlebe er als Naturgewalt: leidenschaftlich oder kämpfend, aber nirgends könne man „gegenseitiges Verstehen“, „liebende Kommunikation“ der Partner erblicken, es gebe „keine innere Entwicklung im gegenseitigen Durchsichtigwerden, das zugleich ein Sichselbstdurchsichtigwerden“ sei. Strindberg sei ohne Drang zum immer weiter fragenden „Klarwerdenwollen“ ${ }^{27}$ gewesen.

Neben Kranken wie Strindberg gebe es Schizophrene ganz anderen Typs. Jeder Psychiater, der nicht nur Symptome jage, sondern sich philosophisch der Existenz annehme, mache eine spezifische Erfahrung (meist nur bei akut Schizophrenen im ersten Schub). Es sei, als ob sich diesen Kranken eine „metaphysische Tiefe offenbare“, Schaudern, Seligkeit errege, um sich im kognitiv defizitären Endzustand zu schließen. Die Lebensführung dieser Kranken werde unbedingter, leidenschaftli-

\footnotetext{
25 In der Skizze zu Jaspers’ Philosophie lehne ich mich eng an: Wolfgang Schüßler: Jaspers zur Einführung. Hamburg 1995, an.

${ }^{26}$ Karl Jaspers: Psychologie der Weltanschauungen. Berlin ${ }^{2} 1922$, S. 1.

27 Jaspers: Strindberg und Van Gogh (wie Anm. 21), S. 68, S. 74.
} 
cher, dämonisch. Wie ließ sich das verstehen? Jenseits der Realität existiere ja Sein, der ewige Geist, der sich in der Zeit in Gefühlen offenbare. Diese Kranken stünden dem Absoluten nahe. Bei Gesunden gedämpft, geordnet, zielgerichtet, bringe sich dies Dämonische hier „zum Durchbruch“; das sei nicht pathologisch, der Geist stehe „außerhalb des Gegensatzes gesund-krank“ (wie er ja auch über jeder Subjekt-Objekt-Spaltung stand). Die Psychose sei Bedingung dieses Durchbruchs, als ob „die Seele aufgelockert würde“; verstehen ließ sich das nur an einzigartigen Menschen, nur in „überragenden, denkkräftigen“, kreativen und „innerlich konsequenten Gestalten“ werde „das Eigentliche der feindlichen Mächte“ deutlich. ${ }^{28}$ Daher behandelte Jaspers Hölderlin und van Gogh, Weltanschauungsund Existenzmodellathleten: Die Psychose „ergriff eine Existenz“, wo sie etwas zum Vorschein brachte, was sonst nicht manifest geworden wäre. Van Goghs Bilder konnten dem Betrachter, dem die „Existenz der schizophrenen Welt zu den wesentlichen, erschütternden Tatsachen des Daseins gehört“ - so Jaspers - etwas begreifbar machen, was andere Kranke nicht zeigten. Die schizophrene Atmosphäre habe hier einen „spezifischen Charakter“, „wesentliches Moment im Kosmos des Geistes“. In der Schizophrenie würden Erfahrungen gemacht, die es vorher so nicht gab. Hier gediehen „neue Kräfte“, die „selbst geistig, weder gesund noch krank" waren.

Wie Mette schätzte auch Jaspers Hölderlins Produktion bis 1806. Hier grob psychiatrische Kategorien anzuwenden, war in Jaspers Sicht unfruchtbar, vielmehr erhellten Eigenschaften dieses Werks das Wesen des Schizophrenen. In der Krankheit habe sich Hölderlins Dichtung nicht mehr an die Realität gewandt, sondern setze sich in eine „zeitlose Welt“, erwachsen aus der „Spannung zwischen erschütternden Gesichten und energischer ordnender Kraft“. Hölderlins mythische Weltanschauung aus gesunden Tagen (Harmonie zwischen Mensch und Natur, Griechentum und dem Göttlichen) sei durch die Psychose erfüllter geworden. Er habe sich seiner Welt genähert, sich von der empirischen Welt und den Menschen entfernt. Dieser früh angelegten Entwicklung sei die Schizophrenie entgegengekommen. Bis 1806 hätten auflösende Kräfte des Prozesses mit dem disziplinierenden Willen, der „Zusammenhalt, Ordnung, Ganzheit“ suchte, gerungen das Entstandene sei einzigartig. Nach 1806 habe die Krankheit Disziplin und Können zerbröckelt. Jaspers kannte nur einen vergleichbaren Fall, der noch stärker seinem Ideal einer erfüllten Existenz entsprach: van Gogh. Dieser habe aufs Wesentliche gezielt, den Sinn der Existenz. Er verkörperte für Jaspers eine Weltanschauung, bei ihm seien „Persönlichkeit, Handeln, Ethos, Existenz und künstlerisches Werk in ungewöhnlichem Maße“ eine Ganzheit gewesen.

Jaspers griff weiter aus, verband Diagnose und Zeit. So wie die Hysterie Affinität zum Geist vor dem 18. Jahrhundert gehabt habe, so passe die Schizophrenie zur Jetztzeit. Der Geist schaffe sich Gestalten unter den ihm gemäßen „psychologisch-causalen Bedingungen“. Die Schizophrenie zeige „verborgene Gründe alles Daseins“, für Gesunde ein „Appell an die eigene Existenz“. Heute sei man „in

${ }^{28}$ Ders.: Autobiographie (wie Anm. 8), S.17, S. 125. 
den letzten Fundamenten des Daseins gelockert“, die Zeit dränge zur Besinnung auf letzte Fragen: „Wir sind“ durch die Lage der Kultur „offen geworden für fremdeste Dinge, sofern sie uns echt und existentiell erscheinen“. ${ }^{29}$

Auch gegen Jaspers lassen sich Einwände finden. So bleibt beispielsweise seine Integration „des“ Schizophrenen in Wesen und Begrifflichkeit in seiner nicht immer präzis fassbaren Seins-Philosophie unklar.

\section{Öffentlichkeit(en) I}

Hängen „Vieldeutigkeit und Unsicherheit“ des Begriffs „Öffentlichkeit“30 damit zusammen, dass er, wie viele historische Begriffe der Politik nicht verabschiedet, aber zugleich in seinem alten Bedeutungsgehalt nicht mehr recht gebraucht werden kann? ${ }^{31}$ Einige knappe Anmerkungen zu seiner Geschichte sowie zu der einiger verwandter Begriffe (öffentlich; publicus; Publikum; öffentliche Meinung) sind nötig. ${ }^{32}$

Öffentlich meinte ursprünglich: vor aller Augen, allen zugänglich. Im 17. Jahrhundert erhielt öffentlich/publicus den Sinn von staatlich (das auch nicht öffentlich, i.e. gebeim sein konnte) im Gegensatz zu privat. Dadurch bezog sich öffentlich immer auch auf das Politische. Im 18. Jahrhundert kam eine weitere Bedeutungsnuance hinzu. Das sich formierende Bürgertum entwickelte spezifische Formen der Geselligkeit, Publikum meinte nun Lesepublikum, das eine bestimmte soziale Schicht umfasste. Publikum war nicht die bloße Addition von Individuen, sondern ethisch-ästhetischer Richter und Erzieher. Der Einzelne könne in seinem Urteil unsicher sein, das Publikum nicht. Bei ästhetischen Urteilen sollte das Publikum befragt werden, das Kunst besser zu beurteilen vermöge als Kunstrichter. Publikum waren „alle geschmackvolle(n) Leute“.33 Was diese als schön deklarierten, das war schön (diese Öffentlichkeit wird später zur kulturellen, sedimentiert im bürgerlichen Feuilleton). Hinter dieser Sicht standen unter anderem Positionen Shaftesburys, der Geschmacksbildung, ästhetische und moralische Erziehung verband. Ästhetisches Urteilen entsprang einer natürlichen Anlage, die Übung und Erziehung benötigte, um korrekte Kunsturteile fällen zu können und

\footnotetext{
${ }^{29}$ Jaspers: Strindberg und Van Gogh (wie Anm. 21), S. 130.

30 Joachim Westerbarkey: Öffentlichkeit und Nicht-Öffentlichkeit. Thesen, Paradoxien und Folgerungen. In: Peter Szyszka (Hg): Öffentlichkeit. Diskurs zu einem Schlüsselbegriff der Organisationskommunikation. Opladen/Wiesbaden 1999, S. 147-155, hier: S. 147.

31 Niklas Luhmann: Öffentliche Meinung. In: ders: Politische Planung. Aufsätze zur Soziologie von Politik und Verwaltung. Opladen ${ }^{4} 1994$, S. 9-34, hier: S. 9.

32 Meine Darlegungen zur Öffentlichkeit basieren auf der überaus hilfreichen Arbeit von Jürgen Schiewe: Öffentlichkeit. Entstehung und Wandel in Deutschland. Paderborn u. a. 2004, an die ich mich eng anlehne.

${ }^{33}$ Friedrich Just Riedel: Ueber das Publicum. Briefe an einige Glieder desselben. Jena 1768, S. 60.
} 
wahren Geschmack zu entwickeln. Tugend und Schönheit gehörten zusammen. ${ }^{34}$ Erziehung fand in kollektiver Aufklärung statt. Der Ausgang aus selbstverschuldeter Unmündigkeit war für einen Einzelnen schwer, dass sich jedoch ein „Publikum selbst aufkläre“ hingegen „beinahe unausbleiblich““. ${ }^{35}$ Aus dem literarischen wurde mit der Französischen Revolution auch ein politisches Publikum, das nach Legitimierung von Herrschaft fragte. Dazu trug die sich entwickelnde öffentliche Meinung bei. Diese war (so ein Kommentar von 1832) die „sich selbst [...] begreifende Vernunft" eines Volks und Staats. ${ }^{36}$

Die Zeit zwischen 1815 und 1880 lässt sich als Zeit der klassischen bürgerlichen „Öffentlichkeit im Liberalismus“ beschreiben. ${ }^{37}$ Im Vormärz, unter der Repression im Deutschen Bund, wurde Öffentlichkeit emphatisch aufgeladen: Frei zu sein meinte, sich frei aussprechen zu können, um so auf das Gemeinwesen zu wirken. Öffentlichkeit war kein Nebenaspekt für Freiheit und Gerechtigkeit, sondern diese selbst. Deswegen war die Forderung nach Pressefreiheit zentral. Die Antike brauchte keine Presse, so argumentierte der Liberale Carl Gustav Jochmann, denn alle Bürger waren bei den Volksversammlungen anwesend. Bei größeren „Völkervereine[n]“ vertrete jedoch die (Presse-)Öffentlichkeit die Stelle der antiken Volksversammlung, hier finde die (Selbst-)Aufklärung des Gemeinwesens statt. Nicht Menschen sollten herrschen, nur Gedanken, deren Wert durch Vergleich und Widerspruch geprüft werden müsse: „Um dem Kampfe der Gewalten $\mathrm{zu}$ entgehen, müssen wir uns den der Meinungen gefallen lassen, denn“ nur die „Reibung sich bekämpfender Meinungen“ führte zur Wahrheit. ${ }^{38}$

Jochmann parallelisierte griechische Agora und Öffentlichkeit im 19. Jahrhundert. So liegt es nah, das Parlament als Modell von Öffentlichkeit zu sehen: Die "ratio des Parlaments" 39 lag in der Annahme des government by discussion. Spezifisch parlamentarische Institute erhielten nur durch Diskussion und Öffentlichkeit Sinn: Ein „Meinungsaustausch“ sollte stattfinden, der vom Zweck beherrscht werde, das Gegenüber rational von der Wahrheit „zu überzeugen oder selbst überzeugt zu werden.“ Das Parlament war der Ort, „an dem die unter den Menschen verstreuten, ungleich verteilten Vernunftpartikeln sich sammeln und zur öffentlichen Herrschaft" gebracht werden.

${ }^{34}$ Michael G. Gill: Lord Shaftesbury (Antony Ashley Cooper, $3^{\text {rd }}$ Earl of Shaftesbury). In: Edward N. Zalta (Hg.): The Stanford Encyclopedia of Philosophy (Fall 2001 Edition); online zugänglich unter: http://plato.stanford.edu/entries/shaftesbury/ (letzter Zugriff am 7. 10. 2015).

35 Immanuel Kant: Was ist Aufklärung. In: ders.: Was ist Aufklärung. Ausgewählte kleine Schriften. Hamburg 1999, S. 20-28, hier: S. 21.

36 [Johann Georg] Mussmann: Öffentliche Meinung. In: Johann Samuel Ersch/Johann Gottfried Gruber (Hg.): Allgemeine Encyclopädie der Wissenschaften und Künste. Dritte Section. Zweiter Theil. Leipzig 1832, S. 52-54.

37 Peter Uwe Hohendahl: Öffentlichkeit - Geschichte eines kritischen Begriffs. Stuttgart/Weimar 2000, S. 38.

38 Carl Gustav Jochmann: Die unzeitige Wahrheit. Weimar 1976, S. $190 f$.

${ }^{39}$ Vgl. zum Folgenden: Carl Schmitt: Die geistesgeschichtliche Lage des heutigen Parlamentarismus. Berlin ${ }^{3} 1961$. 


\section{Öffentlichkeit(en) II}

Zugänglichkeit für alle, Anwesenheit aller - modernisiert durch Stellvertreter-Fiktionen (Parlament, Medien), dichotom verstandener Wettstreit zweier Parteien, die, herrschaftsfrei und rational, als Ausdruck eines Gemeinwesens, seiner Vernunft, für dieses Gemeinwesen ringen: das sind Attribute und Konnotationen des historischen Öffentlichkeitsbegriffs. Es ist fraglich, ob man diesen Begriff so noch konstruieren kann. Einwände finden sich (zu) leicht:

1. Im 19. Jahrhundert bildeten sich marktbedingte Öffentlichkeit(en): ${ }^{40}$ Buch und Zeitung transportieren als Waren nicht unbedingt Wahrheit, sondern Ideologie.

2. Die veröffentlichte Meinung zerfiel in „politische Segmente“ (Liberalismus, Konservatismus, konfessionelle Medien, proletarische Gegenöffentlichkeit). Öffentlichkeit wurde vom Ort argumentativen Wettstreits zum Ort für Meinung(en). Die Medialität von Medien bedingt

- Pluralität (statt Dichotomie),

- für das Gemeinwesen relevante Themen bleiben nicht öffentlich.

3. Auch die Stellvertreter-Funktion des Parlaments blieb letztlich eine Fiktion: Wenige waren anwesend; die sich ausdifferenzierende Parteienkonstellation löste herrschaftsfreies Räsonieren ab; das Parlament war weder Ort noch Ausdruck einer sich selbst begreifenden Staatsvernunft.

4. Der binäre Code des Politischen beziehungsweise des Parlaments ist Regierung/ Macht versus Opposition/Nicht-Macht. Medien funktionieren anders: Aufmerksamkeit versus Nicht-Aufmerksamkeit. Ist öffentliche Meinung also nur Selektion (vielleicht) relevanter Sachverhalte, „die als Themen etabliert werden und zu denen“ Medien mehrheits- und politikfähige Meinungen produzieren? ${ }^{41}$ Ein Thema wird gesetzt, ein anderes nicht: Aufmerksamkeit ist knapp. Gibt es für diese Auswahlverfahren Regeln? ${ }^{42}$ Oder ist es nur einem Überangebot und daraus resultierendem Ignorieren geschuldet ${ }^{33}$

${ }^{40}$ Hans-Ulrich Wehler: Deutsche Gesellschaftsgeschichte. Bd. 3: Von der „Deutschen Doppelrevolution" bis zum Beginn des Ersten Weltkriegs. München 1995, S. 430.

${ }^{41}$ Klaus Merten: Öffentliche Meinung. In: Axel Görlitz/Rainer Praetorius (Hg.): Handbuch Politikwissenschaft. Grundlagen - Forschungsstand - Perspektiven. Reinbek bei Hamburg 1987, S.331, zit. nach: Schiewe: Öfentlichkeit (wie Anm. 32), S. 195.

${ }^{42}$ Luhmann: Meinung (wie Anm. 31), S. 16f., führt u.a. an: „Priorität bestimmter Werte, deren Bedrohung“ ein Thema macht; „Krisensymptome“ als Signal der Bedrohung des Systems insgesamt; „Status des Absenders einer Kommunikation“; „Neuheit von Ereignissen“.

43 „Von den Büchern, die von der Gründung der Bibliothèque nationale bis 1820 gesammelt wurden, sollen bis heute“ [2013] „über zwei Millionen nie ausgeliehen worden sein“, so Günter Karl Bose: Das Ende einer Last. Die Befreiung von den Büchern. Göttingen 2013, S. 30.1992 wurden angeblich mehr als 100000 Bücher gedruckt, von denen nur ein Viertel von Grossisten geführt wurde, $75 \%$ erschienen also „gleichsam unter Ausschluß von Öffentlichkeit“, so Joachim Westerbarkey: Virtuelle Publizität. Öffentlichkeit als imaginäres Kommunikationssystem. In: Werner Faulstich (Hg.): Konzepte von Öffentlichkeit. 3. Lüneburger Kolloquium zur Medienwissenschaft. Bardowick 1993, S. 83-100, hier: S. 83. 
5. Jochmann glaubte, Presse bilde Wirklichkeit ab; doch durch die Medialität von Medien wird Realität modelliert, gar erzeugt.

Überzeugen diese Kritikpunkte? Ist das die richtige Frage? Hervorzuheben zu sein scheint eher: Heutige Öffentlichkeits-Begriffe transportieren $z u$ selbstverständlich alte Konnotationen.

Diese Kritikpunkte wirken zu selbstevident - womöglich weil sie selbst $z u$ stark auf historische Öffentlichkeits-Begriffe bezogen sind?

Fasst man Öffentlichkeit als Interaktion „von medialer Produktion, Kommunikation und Rezeption“, „systemischen und lebensweltlichen Mustern“, „technologischen Dynamiken und kulturellen Bedeutungen“, „biographischen und sozialstrukturellen Dimensionen“ auf, ${ }^{44}$ nimmt die Dichotomie der Parlaments-Metapher von Öffentlichkeit auf und überträgt diese aus der Sphäre des Politischen auf die Gesellschaft, dann wird was Regierung versus Opposition war System versus Lebenswelt, Technologie versus Kultur, Individuum versus Gesellschaft. Drei fragwürdige Annahmen bleiben dabei implizit:

- moderne Gesellschaften sind Konflikt-Gesellschaften - als wäre nicht alle Gesellschaft Konflikt-Gesellschaft (es gibt Jägervereine und Anti-Jägervereine, die Rehe schützen möchten);

- Pluralität und Konflikt verhalten sich wie Ursache zu Wirkung (doch nur wenige sind Mitglieder in Jäger- oder Anti-Jägervereinen, den meisten sind Rehe egal; sie wissen nichts von der Existenz beider Vereine; sie sind Liebhaber von Singvögeln; die Frage nach Rehen stellt sich ihnen einfach nicht). Moderne Gesellschaften sind plural. Es gibt viele Lebenswelten, Systeme, Systeme-Lebenswelten-Kontakte (und: Nicht-Kontakte): Bloße Pluralität erzeugt keine Konflikte;

- dem Agora-Modell der Öffentlichkeit liegt keine Institutionalisierung, „Verfestigung“ zugrunde: Jeder ist als Einzelner anwesend und kann sein Anliegen vorbringen. Das Parlaments-Modell hingegen suggeriert Institutionalisierung: Regierung als Partei steht gegen Opposition. Diese Konnotation hat Folgen. Der Ausdruck Teilöffentlichkeit ist misslich. Meint er bloß Pluralisierung, bleibt er inhaltsleer. Die Teil-Ganzes-Semantik suggeriert aber, dass sich Teile zum Ganzen, der Gesamtöffentlichkeit, fügen (lassen), die oft in der Politik verortet wird. Überdies wird suggeriert, dass Teile ,verfestigt' sind, also Öffentlichkeiten institutionalisiert sind.

Sicher gibt es Konflikte, Aufmerksamkeitsschwellen werden überschritten (und wieder unterschritten), etwas wird öffentlich. Manchmal institutionalisieren sich Teilöffentlichkeiten mebr oder minder mit Publikationsorganen spezifischer Reichweite und mit einem spezifischen Publikum (psychiatriekritische, politischparlamentarische, psychiatrische, literarisch-künstlerische Öffentlichkeit). Fasst man (Teil-)Öffentlichkeit in diesem Sinne auf, dann wäre „psychiatriekritische

${ }^{44}$ So hat es Heinz-Peter Schmiedebach in seinem Exposé zur Tagung vorgeschlagen. 
Bewegung versus Psychiatrie“ wie „Opposition versus Regierung“, „kritische Öffentlichkeit versus Macht“. Dann ließe sich nach der Rolle/Funktion der Psychiatrie (statt: Funktionen), und nach der immer wieder verschobenen „Bedeutung von Wahnsinn“ (statt: Bedeutungen) fragen, wobei es dann dichotom um das Verhältnis einer Fachdisziplin einerseits zu anderen gesellschaftlichen Gruppen andererseits ginge. Wird diese begriffliche Präformierung der semantischen Komplexität des Verhandelten gerecht? Sicher auch. Doch mir scheint, dass die sich daran anknüpfenden Fragen - von denen hier nur eine kleine Liste aufgeführt sei: Wie werden neue öffentliche Räume durch Wahnsinns-Diskurse konzipiert? Wie wurde psychiatrisches Wissen wo rezipiert? Welche psychiatrischen Inhalte zirkulierten zwischen Psychiatern, Medien und Laien? Welche Grenzarbeiten wurden von wem wie vorgenommen? Welche Akteure beförderten welche Strategien? Wo ist Bemächtigung erkennbar? Was erzeugte in Kunst und Literatur Resonanzen? Konstituierten diese Konflikte eine neue Lesart des Wahnsinns? - , so relevant sie auch sind, bei einer anderen Sicht auf Öffentlichkeit(en) verändert werden könnten.

\section{Beispiel, Semantik, Mikroöffentlichkeit}

Geht man von den hier vorgestellten Beispielen - mithin: Inhalten, dem Gebrauch von Schizophrenie-Semantiken - aus, dann handelt es sich bei ihnen um Mikrooder fragmentarische Öffentlichkeiten. Es sind Semantiken, die an Semantiken anschließen und ihrerseits Semantiken erzeugen. Etwas ist öffentlich, wenn es veröffentlicht ist; das ist es, wenn zwei über etwas reden ${ }^{45}$ oder wenn es publiziert ist. Was gesagt beziehungsweise publiziert wurde, ist ein Öffentlichkeitsangebot, ein Angebot, das von unspezifizierten Öffentlichkeiten wahrgenommen, rezipiert und verwendet werden kann. Der Sender hat Absichten (die sowohl unklar als auch unrealisiert bleiben können). Öffentlichkeit wäre ein virtueller semantischer flow: Man kann ihn wahrnehmen (zum Beispiel mittels einer Rezension), ignorieren oder verwenden.

Ergeben sich aus dieser Sicht Ergänzungen zu historisch eingetrübten Öffentlichkeitsbegriffen? Anders gefragt: Was fällt bei den Mikroöffentlichkeiten auf?

1. Zugänglich waren alle drei Bücher, sie waren veröffentlicht. Aber wer war wo anwesend, trug sein Anliegen vor und wurde bemerkt? Als Beispiel soll Mettes Monografie (als veröffentlichte Öffentlichkeit) dienen. Die Wortkunsttheorie hatte eine "Sonderstellung“ im Expressionismus; ihre Streubreite war gering. Bei den Sturm-Lesungen fanden sich 30 bis 100 Zuhörer als festes literarisches Publikum ein. ${ }^{46}$ Schnell erhöht wurde die Streubreite durch eine Rezension in

\footnotetext{
${ }^{45}$ So Westerbarkey: Publizität (wie Anm. 43), S. 84: „Elementare Öffentlichkeit bleibt zumeist publizistisch geheim."

${ }^{46}$ Kurt Möser: Literatur und die „Große Abstraktion“. Kunsttheorien, Poetik und „abstrakte Dichtung“ im „STURM“ 1910-1930. Erlangen 1983, S. II, S. 281.
} 
der „Frankfurter Zeitung“. ${ }^{47}$ In der Zunft war die Wahrnehmung gering und bezog sich nicht auf die Wortkunst. ${ }^{48}$ Das heißt, Mettes Monographie wurde wahrscheinlich im Sturm-Kreis wahrgenommen, im Feuilleton und in der Psychiatrie.

2. Die Akteure in meinem Beispiel sind alle Psychiater. Aber waren sie rollenkonform anwesend? Agierten sie als Psychiater, die sich (für die Psychiatrie) anderer Felder bemächtigen wollten? Das ließe sich nur von Lange sagen. Jaspers und Mette spielten andere Sprachspiele. Alle drei benutzten veröffentlichte, das heißt im semantischen flow als Angebot vorhandene Schizophrenie-Semantiken, veränderten diese und machten daraus wieder ein Angebot. Hier ging es nicht um Psychiatriekritik versus Psychiatrie, Laien versus psychiatrisches Priestertum. Diese Dichotomien sind hier irreführend, weil die Situationen viel komplexer waren. So ist zum Beispiel oft nicht klar, ob sich jemand an eine spezifische, vielleicht schon institutionalisierte Öffentlichkeit wandte (oder kurzfristig eine geschaffen wurde). Das wird deutlicher, wenn man nach den $\mathrm{Ab}$ sichten Mettes und Jaspers' fragt. Ihre Publikationsstrategien geben darauf Hinweise. Mettes Buch, ursprünglich eine Dissertation, erschien im Dion-Verlag, der von Alexander Mette und vom ebenfalls zum Sturm-Kreis zählenden Kurt Liebmann, ${ }^{49} 1925$ zur „Verbreitung expressionistischer Dichtung“, Kunst und Kunsttheorie gegründet worden war. ${ }^{50}$ Mettes Buch segelte unter der Flagge des Sturm-Expressionismus zur Verbreitung dieser Kontrebande. Jaspers Buch erschien zuerst in der Reihe der „Arbeiten zur angewandten Psychiatrie“, „unter Mitwirkung“ unter anderem von ihm und Erwin Stransky herausgegeben von Walter Morgenthaler. Stransky hatte 1918 ein monströses Programm angewandter Psychiatrie formuliert: ${ }^{51}$ Psychiater besäßen überlegene Kenntnisse der Individual-, Gruppen- und Völkerpsychologie, daher seien sie an zentraler Stelle verantwortlich für Gesellschaftsschutz und Rassenhygiene: „die Söhne der bloß beratenden Sachverständigen von heute werden die Richter und Führer der Menschheit von morgen sein“; sie hätten das Ziel vor Augen, alles und alle in den „Hafen der Menschenhöherzucht hineinzusteuern, darin der Arzt der sicherste Lotse ist“. Stranksy träumte von biologistischen Philosophenkönigen in einer Stransky-Plato'schen Diktatur - das war nicht Jaspers’ Interesse. Jaspers interessierte sich für seine eigene Philosophie, folgerichtig erschien die

\footnotetext{
47 Walter Benjamin: Zwei Bücher über Lyrik. In: ders: Gesammelte Schriften. Bd. 3: Kritiken und Rezensionen. Hg. von Hella Tiedemann-Bartels. Frankfurt a. M. 1991, S. 162-166.

${ }^{48}$ Vgl. als Beispiel: Rudolf Treichler: Die seelische Erkrankung Friedrich Hölderlins in ihren Beziehungen zu seinem dichterischen Schaffen. In: Zeitschrift für die gesamte Neurologie und Psychiatrie 155 (1935), S. 40-144; er erwähnt Mette sieben Mal, die Wortkunst interessiert ihn nicht.

49 Markus Krebs: Vom Expressionismus zum Geiste Lessings Kurt Liebmann (1897-1981) (= Signum. Blätter für Literatur und Kritik, Sonderheft 3). Dresden 2001.

${ }^{50}$ Kurt Liebmann/Alexander Mette: Zur Geschichte des Dion-Verlages. In: Marginalien 38 (1978), S. 65-71, hier: S. 65.

51 Vgl. zum Folgenden: Erwin Stransky: Angewandte Psychiatrie. Motive und Elemente zu einem Programmentwurf. In: Archiv für Psychiatrie und Nervenkrankheiten 74 (1918), S. 22-53.
} 
zweite Auflage seines Buchs in den von ihm herausgegebenen „Philosophischen Forschungen“ ${ }^{52}$.

3. Und wie verhält es sich mit dem Ringen um das beste Argument, um Wahrheit und um herrschaftsfreie Ratio? Das setzt voraus, dass die Beteiligten zuhören, dann vergleichen und zustimmen oder widersprechen, wie es Jochmann formulierte. Anders gefragt: Wie wurden Botschaften rezipiert? Grundsätzlich gilt: Medienwirkungsforschung ist mühselig und stochert im Nebel. ${ }^{53}$ Das sei kurz genauer an der Mikroöffentlichkeit Lange-Jaspers-Mette beschrieben. Jaspers las Lange, Mette Jaspers und Lange. Wie waren die Medienwirkungen? Mette postulierte, Jaspers habe gezeigt, dass der Zustand mancher Kranker durch tiefes Erleben, weltanschauliche Erregung gekennzeichnet sei. Weltanschauliche Erregung hieß bei Mette „Expressionismus“, bei Jaspers „Metaphysik“, er verabscheute den Expressionismus. Wie soll man das beschreiben? Kann man hier überhaupt von Rezeption sprechen? Oder ist das Missachtung der Position Jaspers'? Verblieb Mette quasi autistisch in seinen eigenen Interpretationskategorien und benutzte Jaspers nur für sein eigenes Verständnis? Jaspers fand Langes Buch psychiatrisch gut, doch fand er ihn zu wertend. Jaspers meinte, man könne davon absehen. Sollte man es also ignorieren, nicht ernstnehmen?

\section{Schluss}

In diesem Aufsatz wurde die Verwendung von Schizophrenie-Semantiken untersucht. Jeder Autor bastelte mit, aber nicht primär an diesem Konzept. Jaspers brachte Sein, Existenz, Weltanschauung und Schizophrenie, Geschichtsphilosophie (in der Denkfigur des „Zeitgeists“), Ahistorie (in der Figur des Seins) und Krankheitssymptomatologie in ein komplexes Bedeutungsgefüge, das mehr und anderes ist, als individuelle Psychopathologie. Lange transferierte die Diagnose „Schizophrenie“ ebenfalls, machte einen bestimmten Gebrauch von ihr. Er schien ihren Bedeutungsgehalt zu bestätigen. Doch der Transfer veränderte die Arten kommunikativer Anschlussfähigkeit(en) und öffnete das, was als Schizophrenie bezeichnet wurde, für andere semantische Netzwerke und Anschlüsse. Lange griff in die Semantik der Germanistik/Poetologie ein: Wer schreibt wie Hölderlin (schizophren-defekt), erzeugt Nicht-Ästhetisches - das ist eine Aussage darüber, was das Poetische ist. Mette sah das ganz anders. Er fand kommunizierende Röhren zwischen Krankheit und Kunst, Erhellung der Schizophrenie durch Expressionismus, Rechtfertigung der Wortkunst durch Schizophrenie. Dauernd wurde die Semantik umgemodelt durch Gebrauch.

52 Karl Jaspers: Strindberg und Van Gogh. Versuch einer pathographischen Analyse unter vergleichender Heranziehung von Swedenborg und Hölderlin. Berlin ${ }^{2} 1926$.

${ }_{53}$ Vgl. dazu als guten Überblick: Wolfgang Schweiger: Grundlagen: Was sind Medienwirkungen? Überblick und Systematik. In: ders./Andreas Fahr (Hg.): Handbuch Medienwirkungsforschung. Wiesbaden 2013, S.15-37. 
Das Durchspielen dieses Beispiels konnte Ergänzungen zu bisherigen Verständnissen von Öffentlichkeit liefern. Demnach ist das, was veröffentlicht ist, ein Öffentlichkeitsangebot. Jeder der drei machte ein Angebot, das genutzt werden konnte, somit wurden immer neue Öffentlichkeiten (als Kommunikationsangebot, als Semantik) erzeugt. Semantischer flow schaffte Öffentliches, modulierte es, wurde nicht dort rezipiert, wo er wahrgenommen werden sollte oder sogar gar nicht. An erster Stelle stand also der Gebrauch des Begriffs Schizophrenie, und damit der durch Pragmatik umkodierte Sinn. Jeder bemächtigte sich des Begriffs für heterogene Zwecke, die Deutungsressource wurde gebraucht, wieder eingespeist in andere Kommunikationen, unter Umständen erneut als (veränderter) semantischer flow im Öffentlichen, in Öffentlichkeit(en) in die Fachdisziplin zurückgeleitet. - Das hier präsentierte Beispiel ist in dieser Sicht dann auch nur ein Beispiel dafür, was Öffentlichkeit(en) auch bedeuten könn(t)en.

\begin{abstract}
Ever since the disease pattern of dementia praecox/schizophrenia was developed at around 1890/1900, there has never been a completely specified semantic of the concept. Terms and theory were repeatedly employed in different contexts with different purposes in mind. Using three examples, the different semantic uses of the term schizophrenia are scrutinized, constantly dealing with the opus and, at the same time, madness of Friedrich Hölderlin (1770-1843), who came down heavily with a psychological disease after the year 1800. In 1909, Wilhelm Lange-Eichbaum penned a pathography on Hölderlin's case; Karl Jaspers dealt with his case in 1922, as did Alexander Mette in 1929. Following this, the term of 'the public' is outlined and several points of criticism concerning the term's definition are presented. On the basis of the examples mentioned above, the author attempts to develop supplements to the traditional definition of 'the public'.
\end{abstract}

